Internat. J. Math. \& Math. Sci.

Vol. 23, No. 12 (2000) 865-871

S0161171200003215

(C) Hindawi Publishing Corp.

\title{
ON THE NUMERICAL TREATMENT OF THE CONTACT PROBLEM
}

\author{
ABDALLAH A. BADR
}

(Received 13 April 1999)

\begin{abstract}
The problem of the contact of two elastic bodies of arbitrary shape with a kernel in the form of a logarithmic function - which is investigated from Hertz problem-is reduced to an integral equation. A numerical method is adapted to determine the pressure between the two surfaces under certain conditions.
\end{abstract}

Keywords and phrases. Hertz problem, integral equation, orthogonal polynomials.

2000 Mathematics Subject Classification. Primary 45B05.

1. Introduction. Many problems of mathematical physics, theory of elasticity, viscodynamics fluid and mixed problems of mechanics of continuous media reduce to a Fredholm integral equation with continuous or discontinuous kernel. Integral equation containing singular kernel appears in studies involving airfoil [3], fracture mechanics contact [18] radiation and molecular conduction [6] and others. Over the past thirty years, substantial progress has been made in developing innovative approximate analytical and purely numerical solution to a large class of Fredholm integral equation with singular kernel. Since the theory of singular integral equations developed by Muskhelishvili [8] has assumed various technique and has increasing important applications in different areas of science. For this aim, many different methods are established by Tricomi [16], Popov [15], Green [10] and others for obtaining the solution of the integral equations analytically. Since closed form solution to these integral equations are generally not available, great attention has been focused on the numerical treatment. The interested reader should consult the fine exposition by Golberg [8], Linz [11], Atkinson [4], Delves and Mohamed [5]. Since the Fredholm integral equation of the second kind with Cauchy kernel plays an important rule in applied mathematics and physics, so many different numerical solutions are obtained. For example in [7] Gerasoulis used a piecewise quadratic polynomials in the solution of the singular integral equation. As the same way of Gerasoulis, Miller and Keer [12] obtained the solution of the integral equation with Cauchy kernel. In [17] Venturino used Galerkin method to obtain the singular integral equation of the second kind with Cauchy kernel. In [6] Frankel used a Galerkin approach for solving the integrodifferential equation with Cauchy kernel.

In this paper, a numerical method is used to obtain the potential function of a Fredholm integral equation of the second kind with Cauchy kernel. Firstly, we remove the singularity, secondly the solution is expanded in terms of the orthogonal polynomials (we consider the Legendre's polynomial as an example). The solution of the problem reduces to the solution of a linear system. At the end, we give a numerical application to test our method. 
2. Formulation of the contact problem. Consider the semi-symmetric problem [14], when the tangent force, $t(x)$, is related with the normal pressure, $p(x)$, in the contact region of the two surfaces, by the relation

$$
t(x)=k P(x) .
$$

Also, the normal stress, $\tau_{x y}$, with the tangent stress, $\sigma_{y}$, satisfy the relation

$$
\tau_{x y}=k \sigma_{y},
$$

where $k$ is the friction coefficient.

For the displacement components $v_{i}^{*}(i=1,2)$ in the $y$-direction, we have the relation [15]

$$
\frac{d v_{1}^{*}}{d x}=\frac{t(x)}{G_{1}}, \quad \frac{d v_{2}^{*}}{d x}=\frac{t(x)}{G_{2}},
$$

where $G_{1}$ and $G_{2}$ are the displacement compressible materials of two surfaces $f_{1}(x)$ and $f_{2}(x)$, respectively.

It is known that [3] such problem reduces to the following integral equation:

$$
\begin{gathered}
k_{1} \frac{G_{1}+G_{2}}{G_{1} G_{2}} \int_{0}^{x} \phi(t) d t+\left(\nu_{1}+v_{2}\right) \int_{1}^{1} k\left(\frac{x-y}{\lambda}\right) \phi(t) d t=\delta-f_{1}(x)-f_{2}(x), \quad \lambda \in[0, \infty], \\
k(t)=\frac{1}{2} \int_{-\infty}^{\infty} \frac{\tan h u}{u} e^{i u t} d u
\end{gathered}
$$

under the condition

$$
\int_{-1}^{1} \phi(y) d y=p<\infty, \quad \phi(-1)=\phi(1)=0, \quad(p \text { is constant }),
$$

where $\phi(t)$ is the unknown potential function which is continuous through the interval of integration $[-1,1]$, the contact domain between the two surfaces $f_{i}(x)(i=1,2)$, $\delta$ is the rigid displacement under the action of a force $P, k_{1}$ is a physical constant, $k(t)$ is the discontinuous kernel of the problem with singularity at the point $x=y$, and $v_{i}=\left(1-\mu_{i}^{2}\right) /\left(\pi E_{i}\right)(i=1,2)$ where $\mu_{i}$ are the Poisson's coefficients and $E_{i}$ are the coefficients of Young.

As in [15], the kernel can be written in the following form

$$
k(t)=\frac{1}{2} \int_{-\infty}^{\infty} \frac{\tan h u}{u} e^{i u t} d u=-\ln \left|\tan h \frac{\pi t}{4}\right| .
$$

If $\lambda \rightarrow \infty$ and the term $(x-y / \lambda)$ is very small, so that it satisfies the condition $\tan h z \simeq z$, then we have

$$
\ln \left|\tan h \frac{\pi t}{4}\right|=\ln t-d \quad\left(d=\ln \frac{4 \lambda}{\pi}\right) .
$$

Hence, equation (2.4) with the aid of equation (2.7) can be adapted in the form

$$
\int_{0}^{x} \phi(t) d t+v \int_{-1}^{1}[-\ln |y-x|+d] \phi(y) d y=f^{*}(x)
$$


where,

$$
v=\frac{\left(v_{1}+v_{2}\right) G_{1} G_{2}}{k_{1}\left(G_{1}+G_{2}\right)}, \quad f^{*}(x)=\frac{\left[\delta-f_{1}(x)-f_{2}(x)\right] G_{1} G_{2}}{k\left[G_{1}+G_{2}\right]} .
$$

Differentiating equation (2.8) with respect to $x$, we have

$$
\phi(x)+v \int_{-1}^{1} \frac{\phi(y)}{y-x} d y=f(x) \quad\left(f(x)=\frac{d f^{*}(x)}{d x}\right) .
$$

Equation (2.10) represents a Fredholm integral equation of the second kind with Cauchy kernel which will be solved under the condition (2.5).

Here $\int$ denotes integration with Cauchy principal value sense. We suppose that $\phi(x)$, $x \varepsilon[-1,1]$ is continuous and satisfies the normality condition

$$
\left[\int_{-1}^{1}|\phi(y)|^{2} d y\right]^{1 / 2} \leq A\|\phi\|_{2}
$$

where \|\|$_{2}$ denotes the $L_{2}$ norm and $A$ is a constant. Moreover, the potential function $\phi(x)$ satisfies the Lipschitz condition with respect to the second argument. Then $\int(\phi(y)) /(y-x) d y$ exists in the Cauchy principal value sense. It is not difficult to prove the continuity and the normality of the integral operator

$$
K \phi=\int_{-1}^{1} \frac{\phi(y)}{y-x} d y .
$$

In the special case $G_{1}+G_{2}=0, f_{2}(x)=0$ we have the Fredholm integral equation of the first kind with logarithmic kernel, under the condition (2.5). Abdou and Hassan [2] used potential theory to obtain the eigenvalues and eigenfunctions of the problem. Also, Abdou and Ezz-Eldin [1] used Krein's method to solve the same problem.

3. Solution of the problem. In this section, we will solve equation (2.10) under condition (2.5). In order to reach to our goal the singularity of the integral of equation (2.10) will be weakened as follows:

$$
\int_{-1}^{1} \frac{\phi(y)}{y-x} d y=\int_{-1}^{1} \frac{\phi(y)-\phi(x)}{y-x} d y+\phi(x) \int_{-1}^{1} \frac{1}{y-x} d y .
$$

The first of two right integrals is regular and it will be evaluated later while the second integral is evaluated in [9], it is equal to $-\log (1+x) /(1-x)$. Therefore equation (2.10) becomes

$$
\phi(x)+v \int_{-1}^{1} \frac{\phi(y)-\phi(x)}{y-x} d y-v \phi(x) \log \frac{1+x}{1-x}=f(x), \quad-1<x<1 .
$$

Assume the unknown function, $\phi(x)$, can be expanded in terms of a series of Legendre's polynomials:

$$
\phi(x)=\sum_{j=0}^{\infty} a_{j} P_{j}(x)
$$


(one may use any other orthogonal polynomials expansion).

From condition (2.5), we obtain $a_{0}=p$, the rest of the coefficients $a_{j}, j=1,2, \ldots$ are to be determined. The Rodrigues' formula of the Legendre polynomial $P_{j}(x)$ of degree $j$ is given by [9]:

$$
P_{j}(x)=\sum_{k=0}^{[j / 2]} \alpha_{k} x^{j-2 k}
$$

where

$$
\alpha_{k}=\frac{(-)^{k}(2 j-2 k) !}{2 j k !(j-k) !(j-2 k) !} .
$$

From which we obtain

$$
\int_{-1}^{1} \frac{P_{j}(y)-P_{j}(x)}{y-x} d y=\sum_{k=0}^{[(j-1) / 2]} \alpha_{k} \sum_{l=0}^{j-2 k-1} x^{l} \int_{-1}^{1} y^{j-2 k-1-l} d y,
$$

and therefore

$$
\int_{-1}^{1} \frac{P_{j}(y)-P_{j}(x)}{y-x} d y=\sum_{k=0}^{[(j-1) / 2]} \sum_{l=0}^{j-2 k-1} \gamma_{j, k, l} x^{l}
$$

where

$$
\gamma_{j, k, l}=\frac{\alpha_{k}\left[1-(-)^{j-l}\right]}{(j-2 k-l)} .
$$

Using equations (3.3) and (3.7), equation (3.2) becomes:

$$
\left(1-v \log \frac{1+x}{1-x}\right) \sum_{j=0}^{\infty} a_{j} P_{j}(x)+v \sum_{j=1}^{\infty} a_{j} \sum_{k=0}^{[(j-1) / 2]} \alpha_{k} \sum_{j=0}^{j-2 k-1} \gamma_{j, k, l} x^{l}=f(x)
$$

for $-1<x<1$.

Multiply both sides of (3.9) by $x^{i-1}$ for $i=1,2, \ldots, N-1, N$, and then integrating the resultant over the interval $[-1,1]$, we get

$$
\begin{aligned}
& \sum_{j=1}^{\infty} a_{j} \int_{-1}^{1}\left(1-v \log \frac{1+x}{1-x}\right) x^{i-1} P_{j}(x) d x \\
& \quad+v \sum_{j=1}^{\infty} a_{j} \sum_{k=0}^{[(j-1) / 2]} \alpha_{k} \sum_{l=0}^{j-2 k-1} \gamma_{j, k, l} \int_{-1}^{1} x^{l+i-1} d x=\int_{-1}^{1} x^{i-l} f(x) d x,
\end{aligned}
$$

the term-by-term integration is justified by the uniform convergence of each of the previous three series of the left side of the previous equation in the interval $[-1,1]$ and

$$
\left|x^{i} P_{j}(x)\right| \leq\left|x^{i}\right| \leq 1, \quad|x|<1 .
$$

For $|x|<1$, we can assume

$$
\log \frac{1+x}{1-x} \simeq 2 x
$$


and so equation (3.10) will take the form

$$
\begin{gathered}
\sum_{j=1}^{\infty} a_{j}\left\{\int_{-1}^{1}(1-2 v x) x^{i-1} P_{j}(x) d x+v \sum_{k=0}^{[(j-1) / 2]} \alpha_{k} \sum_{l=0}^{j-2 k-1} \frac{2\left[1-(-)^{j-l}\right]}{(j-2 k-l)(l+j)} \delta_{l+j-1}\right\} \\
=\int_{-1}^{1} x^{i-1} f(x) d x-2 p\left(\frac{\delta_{i-1}}{i}-\frac{2 v \delta_{i}}{i+1}\right), \quad \text { for } i=1,2, \ldots, N-1, N
\end{gathered}
$$

where

$$
\delta_{c}= \begin{cases}1, & c \text { even } \\ 0, & c \text { odd }\end{cases}
$$

And we will evaluate the integral of the left side of this equation by using the famous Rodrigues' formula. In fact this integral is equal to

$$
\int_{-1}^{1} x^{i} P_{j}(x) d x=\sum_{k=0}^{[j / 2]} \alpha_{k} \frac{2 \delta_{i, j}}{j+i-2 k+1},
$$

where

$$
\delta_{c, d}= \begin{cases}1, & c+d \text { even and } c \geq d, \\ 0, & \text { otherwise. }\end{cases}
$$

From which the solution of equation (2.10) is the solution of the following linear system:

$$
\begin{gathered}
\sum_{j=1}^{\infty} a_{j}\left\{\sum_{k=0}^{[j / 2]}\left(\frac{2 \alpha_{k} \delta_{i-1, j}}{j+i-2 k}-\frac{4 v \alpha_{k} \delta_{i, j}}{j+i-2 k+1}\right)+\sum_{k=0}^{[(j-1) / 2]} \sum_{l=0}^{j-2 k-1} \frac{2 v \alpha_{k}\left[1-(-)^{j-1}\right]}{(j-2 k-l)(l+i)} \delta_{l+i-1}\right\} \\
=\int_{-1}^{1} x^{i-1} f(x) d x-2 p\left(\frac{\delta_{i-1}}{i}-\frac{2 v \delta_{i}}{i+1}\right)
\end{gathered}
$$

for $i=1,2, \ldots, N-1, N$.

If we truncate the infinite series of the left side of the previous linear system to the first $N$ terms, this linear system will take the form

$$
\sum_{j=1}^{N} c_{i j} a_{j}=b_{i}, \quad i=1,2, \ldots, N-1, N
$$

where

$$
c_{i j}=\sum_{k=0}^{[j / 2]}\left(\frac{2 \alpha_{k} \delta_{i-1, j}}{j+i-2 k}-\frac{4 v \alpha_{k} \delta_{i, j}}{j+i-2 k+1}\right)+\sum_{k=0}^{[(j-1) / 2]} \sum_{l=0}^{j-2 k-1} \frac{2 v \alpha_{k}\left[1-(-)^{j-1}\right]}{(j-2 k-l)(l+i)} \delta_{l+i-1}
$$

and

$$
b_{i}=\int_{-1}^{1} x^{i-1} f(x) d x-2 p\left(\frac{\delta_{i-1}}{i}-\frac{2 v \delta_{i}}{i+1}\right)
$$


TABLE 4.1. The coefficients $a_{j}, j=1,2, \ldots, 20$ (ordered in rows).

\begin{tabular}{rrrrr}
\hline-0.796513983 & 1.282960283 & -1.39797130 & 1.456975757 & -1.549072869 \\
\hline 1.479876614 & -1.567379746 & 1.417647537 & -1.559090422 & 1.178337053 \\
\hline-1.392267336 & 2.037120656 & -1.596255523 & -2.924756126 & -50248330647 \\
\hline 8.38415880 & 6.333866610 & 1.704701286 & -0.252825184 & -1.196720603 \\
\hline
\end{tabular}

4. Numerical example. The solution of the integral equation (3.2) depends on the Cauchy kernel and the two surfaces $f_{1}(x)$ and $f_{2}(x)$. We can expand each of these two functions in Macklaurin expansion near $x=0$ where the initial points and the tangent points of the surfaces are in contact with the origin $O$. For that reason, we can assume the function $f(x)$ is a polynomial (see the definition of the function $f(x)$, equation (2.10)).

If we take only the quadratic term in the Maclorian expansion of the function $f_{1}(x)+$ $f_{2}(x)$, then we may assume $f(x)=x$. Also, take $p=0.8, v=0.25$, and $N=10$. The pressure between the two surfaces in this case is given by

$$
\phi(x)=\sum_{j=0}^{10} a_{j} P_{j}(x),
$$

where, $P_{j}(x)$ is Legendre's polynomial of degree $j$ and the coefficients $a_{j}$ are the solution of the linear system (3.18), we used Maple $V$ to solve such system. These coefficients are tabulated in Table 4.1.

\section{REFERENCES}

[1] M. A. Abdou and N. Y. Ezzeldin, Krěn's method with certain singular kernel for solving the integral equation of the first kind, Period. Math. Hungar. 28 (1994), no. 2, 143-149. MR 95i:45001. Zbl 816.45001.

[2] M. A. Abdou and S. A. Hassan, Boundary value of a contact problem, Pure Math. Appl. 5 (1994), no. 3, 311-316. CMP 1343 454. Zbl 829.45004.

[3] V. M. Alexandrov and E. V. Kovalenko, Problems with Mixed Boundary Conditions in Continuous Mechanics, Nauka, Moscow, 1986.

[4] K. E. Atkinson, A Survey of Numerical Methods for the Solution of Fredholm Integral Equations of the Second Kind, SIAM, Philadelphia, Pa., 1976. MR 58\#3577. Zbl 353.65069.

[5] L. M. Delves and J. L. Mohamed, Computational Methods for Integral Equations, Cambridge University Press, Cambridge, New York, 1985. MR 87j:65159. Zbl 592.65093.

[6] J. I. Frankel, A Galerkin solution to a regularized Cauchy singular integro-differential equation, Quart. Appl. Math. 53 (1995), no. 2, 245-258. MR 96b:45002. Zbl 823.65145.

[7] A. Gerasoulis, The use of piecewise quadratic polynomials for the solution of singular integral equations of Cauchy type, Comput. Math. Appl. 8 (1982), no. 1, 15-22. MR 82m:65123. Zbl 467.65071.

[8] M. A. Golberg, The convergence of a collocation method for a class of Cauchy singular integral equations, J. Math. Anal. Appl. 100 (1984), no. 2, 500-512. MR 85i:65172. Zbl 588.65092. 
[9] I. S. Gradstein and I. M. Ryzik, Tablitsy Integralov, Summ, Ryadov i Proizvedenil [Tables of Integrals, Sums, Series and Products], Gosudarstv. Izdat. Fiz.-Mat. Lit., Moscow, 1963. MR 28\#5198.

[10] C. D. Green, Integral Equation Methods, Thomas Nelson \& Sons Ltd., London, 1969. MR 40\#658. Zbl 179.44301.

[11] P. Linz, Analytical and Numerical Methods for Volterra Equations, Society for Industrial and Applied Mathematics (SIAM), Philadelphia, Pa., 1985. MR 86m:65163. Zbl 566.65094.

[12] G. R. Miller and L. M. Keer, A numerical technique for the solution of singular integral equations of the second kind, Quart. Appl. Math. 42 (1985), no. 4, 455-465. MR 87c:65165a. Zbl 593.65094.

[13] N. I. Muskhelishvili, Singular Integral Equations. Boundary Problems of Function Theory and their Application to Mathematical Physics, P. Noordhoff N. V., Groningen, 1953. MR 15,434e. Zbl 051.33203.

[14] _ Some Basic Problems of the Mathematical Theory of Elasticity, Society for Industrial and Applied Mathematics (SIAM), Philadelphia, Pa., 1985.

[15] G. Y. Popov, Contact Problems for a Linearly Deformable Base, Vishcha Shkola, KievOdessa, 1982 (Russian). MR 84d:73004. Zbl 563.73098.

[16] F. G. Tricomi, Integral Equations, Dover Publications Inc., New York, 1985. MR 86k:45001. Zbl 078.09404.

[17] E. Venturino, The Galerkin method for singular integral equations revisited, J. Comput. Appl. Math. 40 (1992), no. 1, 91-103. MR 93h:65170. Zbl 755.65136.

[18] J. R. Willis and S. Nemat-Nasser, Singular perturbation solution of a class of singular integral equations, Quart. Appl. Math. 48 (1990), no. 4, 741-753. MR 92a:45016. Zbl 726.45005 .

BADR: ALEXANDRIA UNIVERSITY, ALEXANDRIA, EGYPT

E-mail address: badrzoo@yahoo.com 


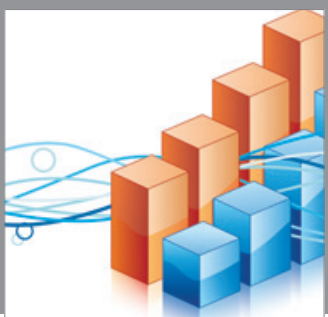

Advances in

Operations Research

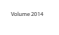

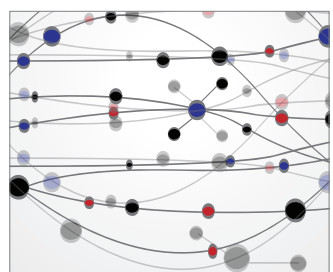

\section{The Scientific} World Journal
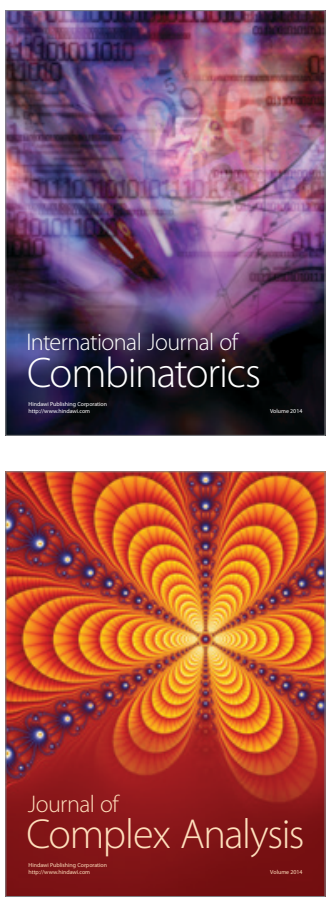

International Journal of

Mathematics and

Mathematical

Sciences
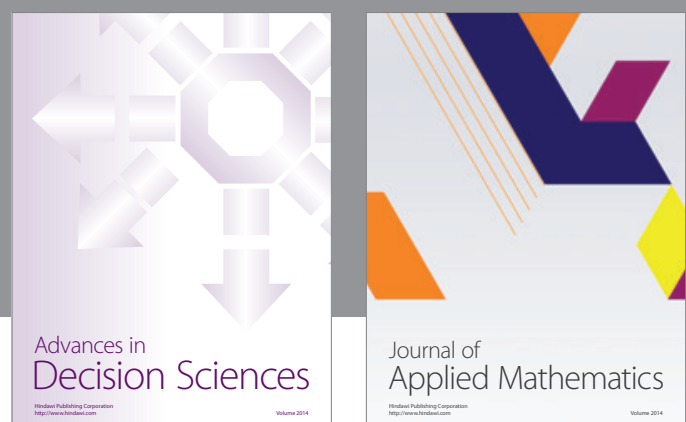

Journal of

Applied Mathematics
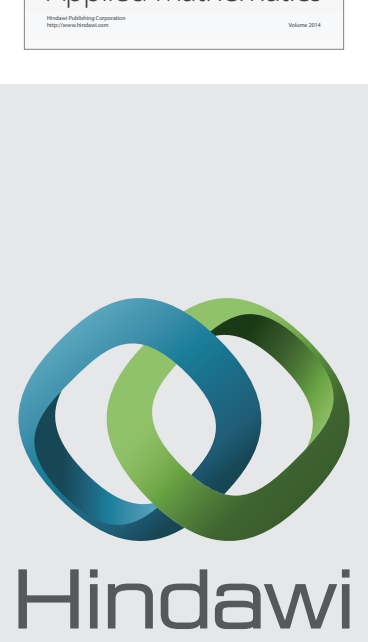

Submit your manuscripts at http://www.hindawi.com
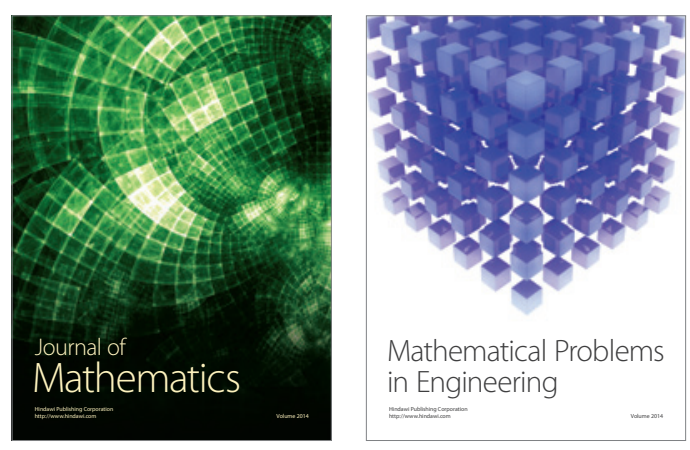

Mathematical Problems in Engineering
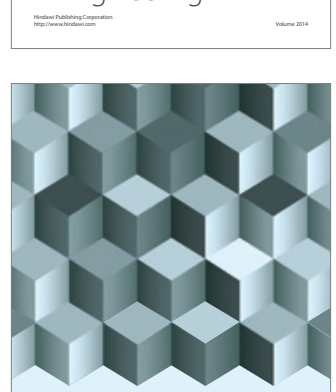

Journal of

Function Spaces
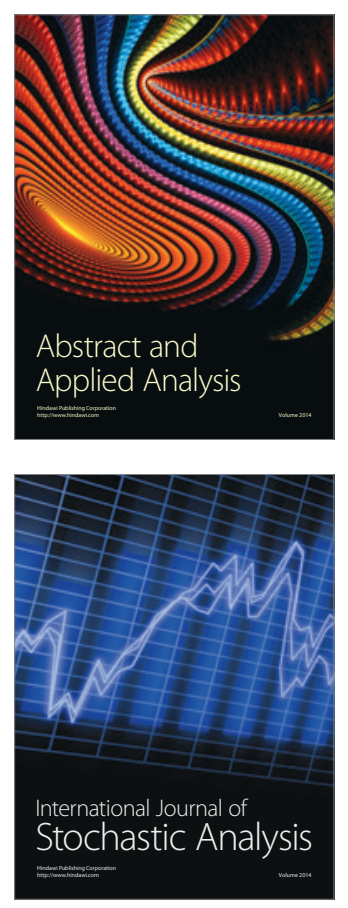

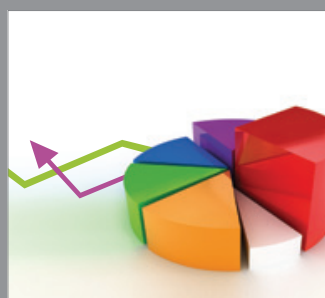

ournal of

Probability and Statistics

Promensencen
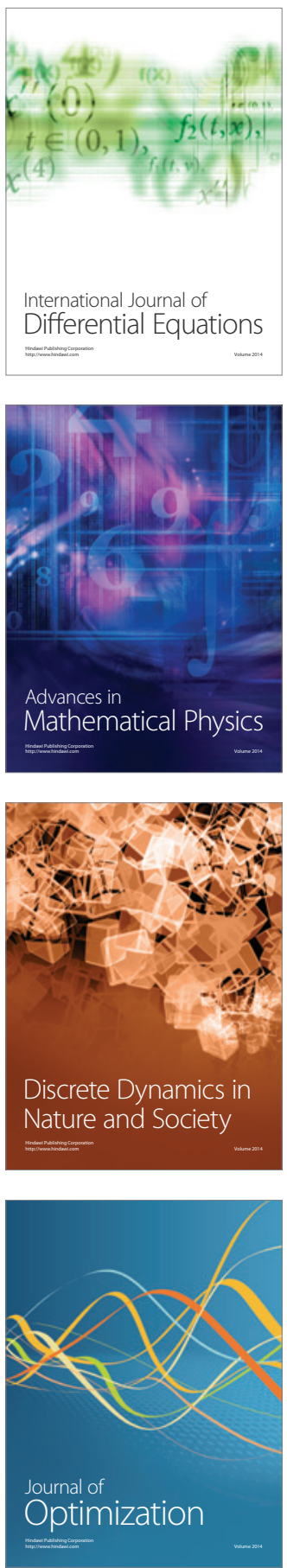